\title{
TID effects in deep N-well CMOS monolithic active pixel sensors
}

\author{
Lodovico Ratti, Member, IEEE, Claudio Andreoli, Luigi Gaioni, Massimo Manghisoni, Member, IEEE, \\ Enrico Pozzati, Valerio Re and Gianluca Traversi, Member, IEEE
}

\begin{abstract}
This paper is devoted to the study of total ionizing dose effects in deep N-well (DNW) CMOS monolithic active pixel sensors (MAPS) for particle tracking fabricated in a STMicroelectronics $130 \mathrm{~nm}$ process. DNW-MAPS samples were exposed to $\gamma$-rays up to a final dose of $1100 \mathrm{krad}\left(\mathrm{SiO}_{2}\right)$ and then subjected to a $100^{\circ} \mathrm{C}$ annealing cycle. Ionizing radiation tolerance was tested by monitoring the device noise properties and its response to charge injection through an external pulse generator throughout the irradiation and annealing campaign. The origins of performance degradation are discussed based on the results from radiation hardness characterization of single transistors belonging to the same CMOS technology and of test diodes reproducing the MAPS collecting electrode structure. Also circuit simulations have been performed to supply further evidence for the proposed degradation mechanisms.
\end{abstract}

Index Terms-MAPS, deep N-well, ionizing radiation, CMOS, analog front-end.

\section{INTRODUCTION}

$\mathbf{M}$ ONOLITHIC active pixel sensors (MAPS) take advantage of the large scale of integration of commercial CMOS processes to integrate both the sensing element and the mixed-signal processing electronics in the same substrate. Their features are exploited in multi-megapixel imagers [1] for consumer and professional applications such as digital still photography and cameras for high definition TV. In the last few years MAPS have been proposed as likely candidates for charged particle tracking at the next generation, high luminosity colliders and B-factories [2], [3]. In fact, as their working principle is based on the collection of charge diffusing in a high resistivity epitaxial layer, they can theoretically be thinned down to a few tens of microns, therefore providing improved momentum resolution. They can also offer higher granularity than standard hybrid pixel detectors, thanks to their very simple readout schemes, which are mostly based on a pixel-level source follower amplifier and a sequential readout architecture. A new approach, which relies on the properties of the triple well structures included in modern CMOS processes, has been recently put forward and led to the design and fabrication of so called deep N-well (DNW) MAPS [4]. In these

Manuscript received December 16, 2008. This work was supported by the Italian Ministry of University and Research through a National Firb Project, contract \#RBAP06L4S5.

Claudio Andreoli, Luigi Gaioni, Enrico Pozzati and Lodovico Ratti are with INFN Pavia and Dipartimento di Elettronica, Università degli Studi di Pavia, I-27100 Pavia, Italy (Phone: +39 0382 985222; fax: +39 0382422583 ; email: lodovico.ratti@unipv.it).

Massimo Manghisoni, Valerio Re and Gianluca Traversi are with INFN Pavia and Dipartimento di Ingegneria Industriale, Università di Bergamo, I24044 Dalmine (BG), Italy. devices, a relatively (as compared to standard three transistor MAPS) large collecting electrode featuring a buried N-type layer is integrated with a classical readout chain for capacitive detectors. This solution was suggested chiefly to comply with the intense data rates foreseen for tracking applications at the future high energy physics (HEP) facilities. Actually, based on the proposed device, the first ever MAPS detectors with pixel-level data sparsification have been designed and produced [5], [6]. Depending on the experiment characteristics and on the position inside the detector, MAPS sensors may be required to withstand a total ionizing dose (TID) which can range from a few hundreds of $\operatorname{krad}\left(\mathrm{SiO}_{2}\right)$ to a few tens of $\operatorname{Mrad}\left(\mathrm{SiO}_{2}\right)$ [7], [8]. This work is concerned with the analysis of ionizing radiation effects on DNW-MAPS fabricated in a $130 \mathrm{~nm}$ CMOS technology with triple well feature. The aim is to assess the suitability of the proposed devices for radiation hard operation. As the ones presented in this paper are the first extensive irradiation tests performed on deep $\mathrm{N}$ well monolithic pixels, the results are also expected to provide some useful hints for more robust design of the future DNWMAPS generations.

\section{DUTS AND TEST PROCEDURES}

The structures under test are included in a chip, called Apsel2T and described in detail in Table I. In all of the tested devices, the signal coming from the sensor (or from an external pulse generator through a $60 \mathrm{fF}$ injection capacitor) is processed by means of the readout chain shown in Fig. 1, including a charge preamplifier and an RC-CR shaper. As anticipated in the introduction, the sensor has been designed by taking advantage of a deep N-well structure. Although not explicitly indicated in the figure, the analog front-end is mostly integrated inside the DNW, which acts as the collecting electrode and features a double junction, one towards the substrate and one towards the internal P-well. Not shown in the figure, a discriminator is used to compare the signal at the shaper output to a preset threshold voltage while a 1-bit memory cell is used to store the hit. The charge preamplifier features a folded cascode scheme [9], where $M_{i n}$ is the input device and $M_{c s}$ is the PMOS current source in the circuit input branch. $C_{F}$ is the preamplifier feedback capacitor (nominally $8 \mathrm{fF}$ ), continuously reset by means of the $M_{F}$ NMOS transistor operated in the deep subthreshold region. The preamplifier output stage is represented by means of an ideal buffer. In the shaping filter, $C_{1}$ is the differentiating input capacitance while $C_{2}$ is the capacitance in the shaper feedback network, which also 


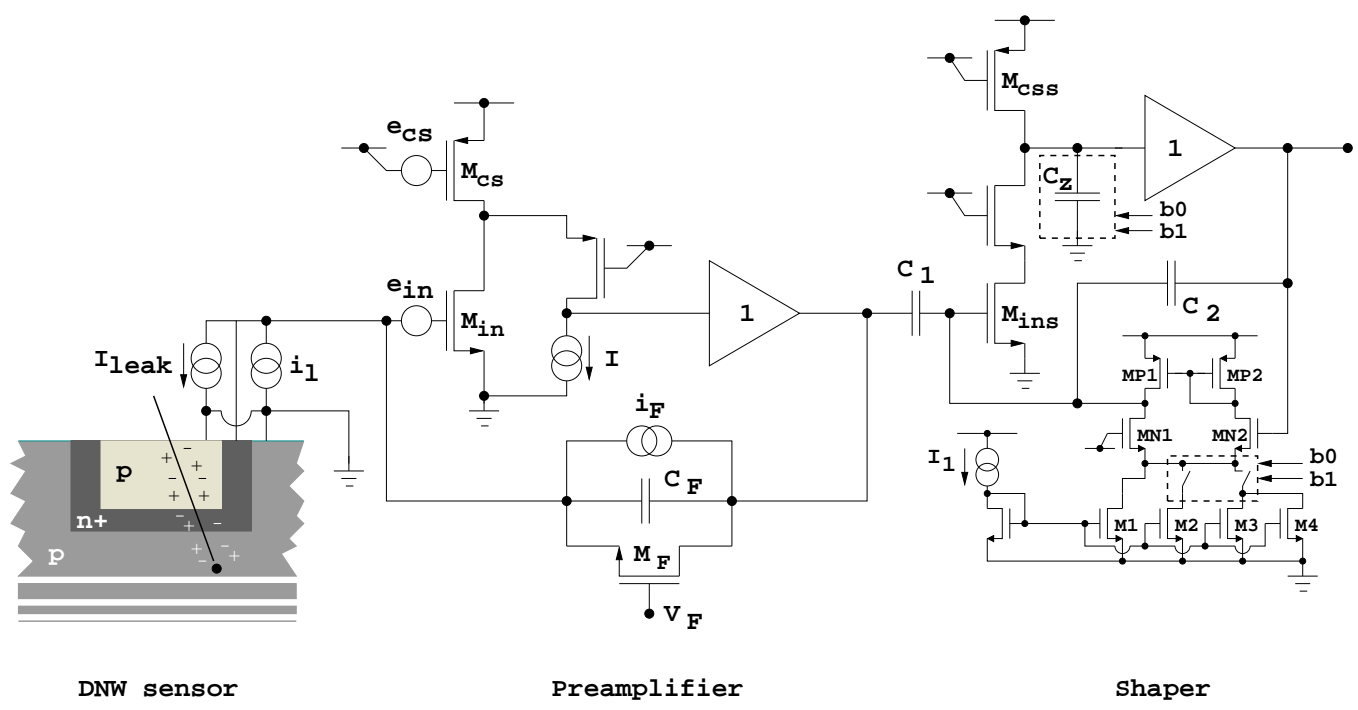

Fig. 1. Schematic diagram of the analog readout chain integrated in the deep $\mathrm{N}$-well monolithic active pixel sensors. A conceptual cross sectional view of the deep $\mathrm{N}$-well sensor is also shown.

TABLE I

APSEL2T CHIP DESCRIPTION

\begin{tabular}{|c|l|}
\hline T1 & $\begin{array}{l}3 \times 3 \text { matrix, all analog outputs accessible, injection } \\
\text { capacitance }\left(C_{i n j}=60 \mathrm{fF}\right) \text { for the central pixel, } \\
900 \mu \mathrm{m}^{2} \text { collecting electrode area }\end{array}$ \\
\hline T2 & $\begin{array}{l}3 \times 3 \mathrm{matrix} \text { three analog outputs accessible, } \\
900 \mu \mathrm{m}^{2} \text { collecting electrode area }\end{array}$ \\
\hline T3 & $\begin{array}{l}3 \times 3 \text { matrix, three analog outputs accessible, injection } \\
\text { capacitance }\left(C_{i n j}=60 \mathrm{fF}\right) \text { for the central pixel, } \\
900 \mu \mathrm{m}^{2} \text { collecting electrode area, shaper MIM } \\
\text { capacitors laid over the readout electronics }\end{array}$ \\
\hline T4 & $\begin{array}{l}3 \times 3 \text { matrix, three analog outputs accessible, injection } \\
\text { capacitance }\left(C_{\text {inj }}=60 \mathrm{fF}\right) \text { for the central pixel, } \\
1500 \mu \mathrm{m}^{2} \text { collecting electrode area }\end{array}$ \\
\hline SRC & $\begin{array}{l}\text { standalone readout channel with injection capacitance } \\
\left(C_{i n j}=60 \mathrm{fF}\right)\end{array}$ \\
\hline
\end{tabular}

includes a transconductor (transistors $M N 1, M N 2, M P 1$ and $M P 2)$. Again, the output stage of the shaper is depicted as an ideal buffer. By acting (through slow control bits bo and b1) on the capacitance $C_{z}$ in the high impedance node of the shaper gain stage and on the transconductor current sources ( $M 1$ to $M 4$, all with identical gate dimensions), the peaking time can be set to $0.5,1$ or $2 \mu$ s [10]. Fig. 1 also includes the main preamplifier noise sources and represents the shaper architecture with some degree of detail. Reference to the figure will be made and its description will be completed in the next sections, when the effect of radiation on the different circuit parts and on the overall front-end performances will be discussed.

Besides the above described MAPS, other devices, fabricated in the same technology as the monolithic sensors under test, have been characterized from the standpoint of radiation hardness. Among them, single NMOS transistors with different gate widths $(\mathrm{W}=1000,600,20,10,0.18 \mu \mathrm{m})$ and lengths $(\mathrm{L}=0.13,0.35,10 \mu \mathrm{m})$, and different kinds of pn junction diodes, including diodes with a deep $\mathrm{N}$-well, double junction structure, as in the DNW-MAPS sensor. In the irradiation tests described in this paper, the DUTs were exposed to $\gamma$-rays from a ${ }^{60} \mathrm{Co}$ source featuring a dose rate of $12 \mathrm{rad} / \mathrm{s}\left(\mathrm{SiO}_{2}\right)$. The final integrated dose, $1100 \mathrm{krad}\left(\mathrm{SiO}_{2}\right)$, was reached through one or more intermediate irradiation steps. The devices under test were also subjected to a $100{ }^{\circ} \mathrm{C}$ annealing cycle for 168 hours. All the DUTs were biased during both the irradiation and the annealing cycles. In particular, DNW-MAPS were biased as they would be in actual applications, diodes were biased with a reverse voltage of $300 \mathrm{mV}$ (like the DNW-MAPS collecting electrode) and NMOS transistors had their gate terminal at $1.2 \mathrm{~V}$ while keeping all the other terminals grounded. It is worth noticing here that no specific radiation hard techniques (e.g., use of enclosed layout transistors or of thin oxides over the junctions) were applied in the design of the collecting electrode or of the processing electronics.

\section{EFFECTS OF IONIZING RADIATION ON DNW-MAPS PERFORMANCES}

The effects of ${ }^{60} \mathrm{Co} \gamma$-rays on DNW-MAPS has been studied by evaluating in which fashion the main device parameters, namely charge sensitivity and equivalent noise charge (ENC) are affected by irradiation. MAPS test results, together with the characterization of single transistors and pn diodes fabricated in the same technology, helped single out the most critical circuit parts and design issues.

\section{A. Charge sensitivity}

In the DNW-MAPS front-end, the shaping stage components have been finely tuned so that the equality

$$
G_{m 0}=\frac{G B P_{0} C_{2}^{2}}{4\left(C_{1}+C_{2}\right)}
$$

holds and a semigaussian, first order unipolar shaping can be achieved [4]. In (1), $G_{m 0}$ is the transconductance of the shaper transconductor and $G B P_{0}=\frac{g_{m, i n s, 0}}{C_{z}}$ is the gain-bandwidth product of the shaper gain stage, with $g_{m, i n s, 0}$ the channel transconductance of the shaper input transistor $M_{i n s}$. The 
subscript ' 0 ' in the previous symbols indicates preirradiation values. If the charge preamplifier provides a step response, perfectly linear in amplitude, to a delta-shaped input pulse, then the charge sensitivity $G_{Q}$ is given by

$$
G_{Q}=\frac{\left|\max \left\{v_{\text {out }, s}(t)\right\}\right|}{Q}=2 \frac{C_{1}}{C_{F} C_{2} e}
$$

where $v_{\text {out }, s}(t)$ is the signal at the shaper output as a response to a $Q$ area input pulse and $e$ is Neper's constant. Exposure to $\gamma$-rays has the effect of decreasing charge sensitivity. This is shown, for instance, in Fig. 2 and Fig. 3 for a standalone readout channel, not connected to the DNW sensor, and for the central pixel of a matrix of the T1 type respectively. Radiation induced changes in the charge sensitivity can be conveniently studied by separately examining the effects taking place in the two main front-end blocks:

- change in the equivalent preamplifier feedback resistance, due to threshold voltage shift in the feedback NMOSFET and to its operating point being modified by the leakage current from the detector (the latter effect is expected to take place only in T1, T2, T3 and T4 type structures,

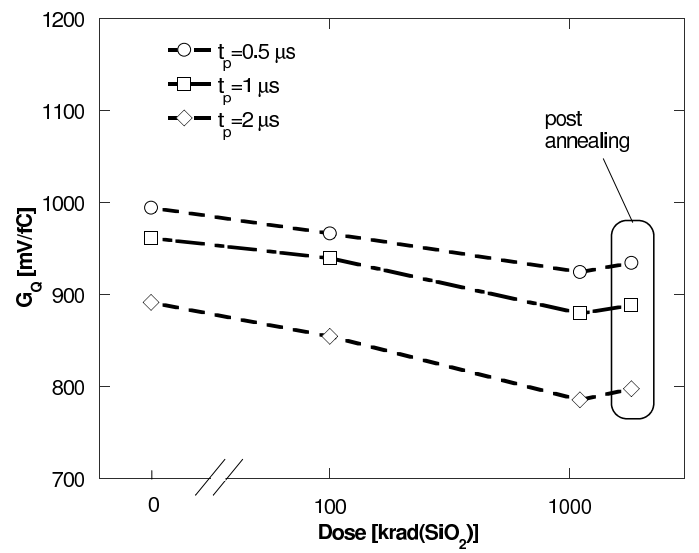

Fig. 2. Charge sensitivity as a function of the total ionizing dose and after the annealing procedure for a standalone readout channel. Data are plotted for the three available peaking times.

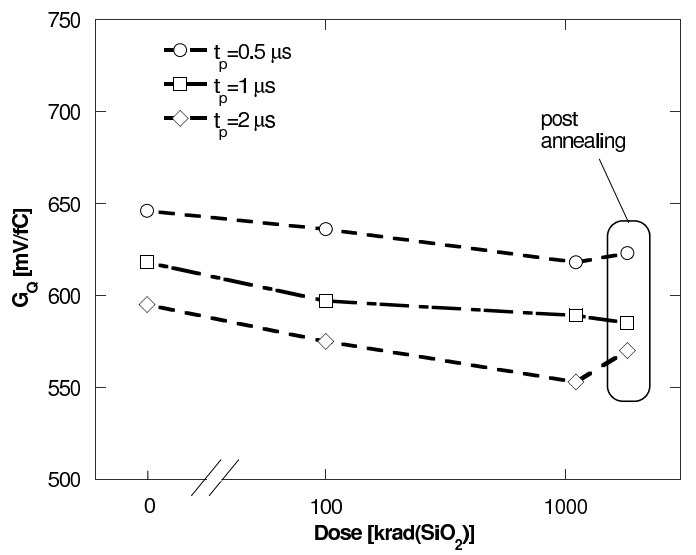

Fig. 3. Charge sensitivity as a function of the total ionizing dose and after the annealing procedure for the central pixel of a MAPS matrix of the T1 type. Data are plotted for the three available peaking times. where the front-end electronics is actually connected to the sensor);

- change in the feedback transconductance and in the gain bandwidth product in the shaping stage.

1) Radiation effects in the charge preamplifier: Radiation induced effects in the charge preamplifier gain can be more effectively evaluated if a finite value is assumed for the characteristic reset time constant $\tau_{F}=\frac{C_{F}}{g_{d s, F}}$, where $g_{d s, F}$ is the output conductance of the preamplifier feedback MOSFET. In an NMOS device operated in the deep subthreshold region, like $M_{F}$, the drain current is given by [11]

$$
I_{D}=I_{D 0} \frac{W}{L} e^{\frac{q\left(V_{G S}-V_{t h, F}\right)}{n k_{B} T}}\left(1-e^{-\frac{q V_{D S}}{k_{B} T}}\right)
$$

where $I_{D 0} \simeq 6 \mu_{n 0} C_{o x}\left(\frac{k_{B} T}{q}\right)^{2}, \mu_{n 0}$ is the zero-bulk-bias electron mobility, $C_{o x}$ is the oxide capacitance per unit area, $W$ and $L$ are the transistor channel width and length respectively, $q$ is the elementary charge, $V_{G S}$ is the source-to-gate voltage, $V_{t h, F}$ is the threshold voltage, $n$ is the subthreshold slope coefficient, $k_{B}$ is the Boltzmann's constant, $T$ is the absolute temperature and $V_{D S}$ the source-to-drain voltage. It can be easily shown that

$$
\frac{\partial g_{d s, F}}{\partial V_{t h, F}}=\frac{\partial}{\partial V_{t h, F}} \frac{\partial I_{D}}{\partial V_{D S}}<0 .
$$

Therefore, a decrease in $V_{t h, F}$ may result in an increase of the output conductance of $M_{F}$ and, in turn, in a decrease of $\tau_{F}$. Although deep submicron CMOS technologies are widely known to feature a high degree of radiation tolerance [12], narrow channel devices $(\mathrm{W}<1 \mu \mathrm{m})$ belonging to the same CMOS generation as the technology used for the DNW-MAPS discussed here were found to undergo the so called RINC (radiation-induced narrow channel) effect, originating from charge trapping in the shallow trench isolation oxides and proven to affect both $\mathrm{N}$ and P-channel devices [13]. A very similar behavior was actually found in irradiated devices from the same $130 \mathrm{~nm}$ process as the DNW-MAPS. This is shown in Table II, displaying the threshold voltage shift $\Delta V_{t h, F}$ for a set of devices with different gate dimensions after exposure to a $1100 \mathrm{krad}\left(\mathrm{SiO}_{2}\right) \gamma$-ray TID and after annealing. The threshold shift, around a few $\mathrm{mV}$ in all the devices with gate width no smaller than $10 \mu \mathrm{m}$, is significantly larger in the narrow channel device, which, by the way, features the same gate dimensions as $M_{F}$. After the $100^{\circ} \mathrm{C} / 168 \mathrm{~h}$ annealing cycle,

TABLE II

THRESHOLD VOLTAGE VARIATION IN IRRADIATED $130 \mathrm{~nm}$ NMOSFETS

\begin{tabular}{l|c|c} 
Gate dimensions & \multicolumn{2}{|c}{$\Delta V_{t h}[\mathrm{mV}]$} \\
\cline { 2 - 3 }$[\mu \mathrm{m} / \mu \mathrm{m}]$ & $1100 \mathrm{krad}\left(\mathrm{SiO}_{2}\right)$ & After annealing \\
\hline $1000 / 0.13$ & 0 & 0 \\
$1000 / 0.35$ & -2 & 0 \\
$600 / 0.35$ & -4 & -1 \\
$20 / 0.13$ & -2 & 0 \\
$20 / 0.20$ & -3 & 0 \\
$20 / 0.35$ & -3 & -1 \\
$10 / 0.35$ & -3 & -1 \\
$10 / 0.13$ & -5 & -2 \\
$\mathbf{0 . 1 8} / 10$ & $\mathbf{- 2 0}$ & $\mathbf{- 3}$
\end{tabular}




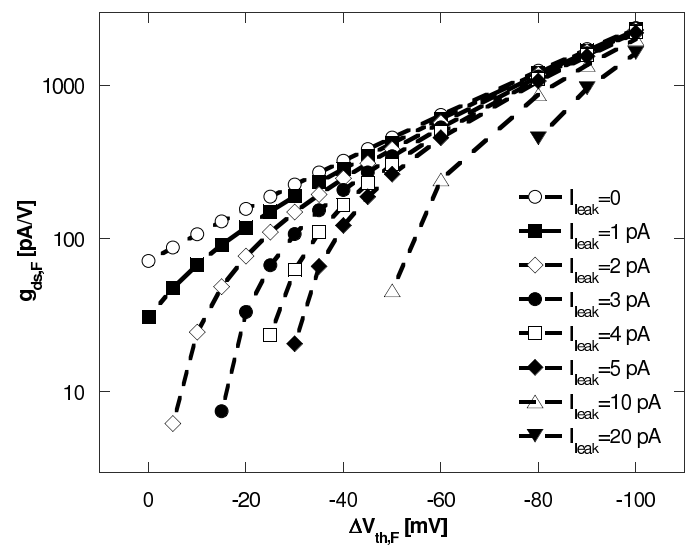

Fig. 4. $M_{F}$ output conductance $g_{d s, F}$ as a function of its threshold shift $\Delta V_{t h, F}$ as obtained from circuit simulations.

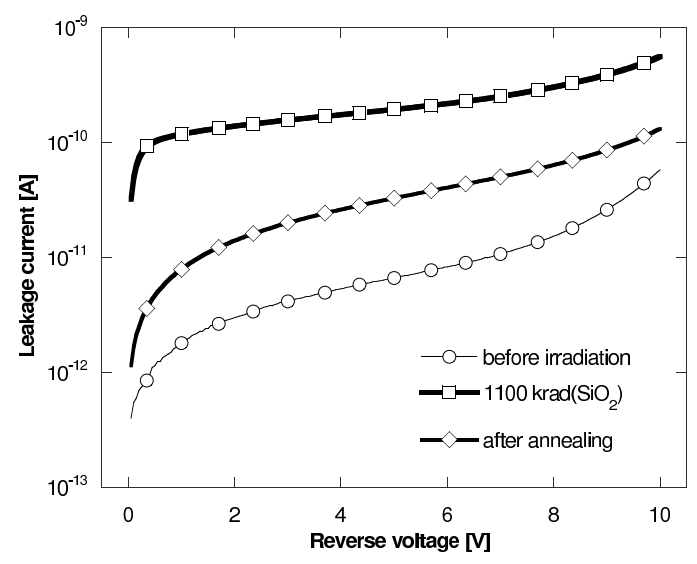

Fig. 5. Leakage current as a function of the reverse voltage in a deep Nwell diode. The preirradiation curve is compared to the ones obtained after exposure to a $1100 \mathrm{krad}\left(\mathrm{SiO}_{2}\right) \mathrm{TID}$ and after the $100^{\circ} \mathrm{C} / 168 \mathrm{~h}$ annealing cycle.

pristine conditions are almost completely recovered by all the devices, including the narrow channel one. The effects of a change in the $M_{F}$ threshold voltage are shown in Fig. 4, where $g_{d s, F}$ is plotted as a function of $\Delta V_{t h, F}$ as it results from circuit simulations. In the same figure, the behavior of $g_{d s, F}$ is also represented for different values of the leakage current $I_{\text {leak }}$ in the DNW sensor. The latter parameter was found to undergo a significant radiation induced change in DNW diode test samples with the same layer structure as the sensor depicted in Fig. 1, though featuring different geometry (circular for the diode, T-shaped for the MAPS collecting electrode) and dimensions (about $30000 \mu^{2}$ area for the diode). The relevant data are displayed in Fig. 5. The increase in leakage current with increasing total ionizing dose, almost two orders of magnitude at $1100 \mathrm{krad}\left(\mathrm{SiO}_{2}\right)$, may be ascribed to the buildup of holes in the field oxide which may extend the space charge region at the diode junction and increase the surface generation current [14]. Combined with the previous mechanism, the creation of trapping states at the $\mathrm{Si} / \mathrm{SiO}_{2}$ interface over the pn junction depletion region may account for a further increase of the surface generation phenomena [15]. The plots in Fig. 4 show a decrease of $g_{d s, F}$ with increasing

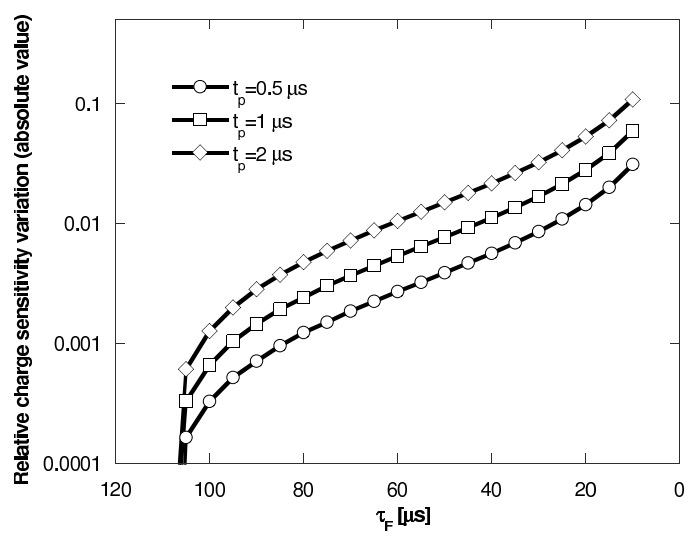

Fig. 6. Computed relative charge sensitivity variation as a function of the preamplifier reset time constant $\tau_{F}$.

TABLE III

RELATIVE GAIN VARIATION IN SRC AND T1 TYPE DEVICES UPON ABSORPTION OF A $1100 \mathrm{krad}\left(\mathrm{SiO}_{2}\right)$ TID

\begin{tabular}{c|c|c|c|c|c}
\multicolumn{2}{c|}{$t_{p}=0.5 \mu \mathrm{s}$} & \multicolumn{2}{c|}{$t_{p}=1 \mu \mathrm{s}$} & \multicolumn{2}{c}{$t_{p}=2 \mu \mathrm{s}$} \\
\hline $\mathrm{SRC}$ & $\mathrm{T} 1$ & $\mathrm{SRC}$ & $\mathrm{T} 1$ & $\mathrm{SRC}$ & $\mathrm{T} 1$ \\
\hline$-7.0 \%$ & $-4.3 \%$ & $-8.5 \%$ & $-4.7 \%$ & $-11.9 \%$ & $-7.1 \%$
\end{tabular}

$I_{\text {leak }}$. It can be actually easily shown that, once $I_{D}=I_{\text {leak }}$ has been set in (3), then

$$
g_{d s, F}=\frac{I_{D 0} q W e^{\frac{q\left(V_{G S}-V_{t h, F}\right)}{n k_{B} T}}}{L k_{B} T}-\frac{I_{\text {leak }} q}{k_{B} T} \Rightarrow \frac{\partial g_{d s, F}}{\partial I_{\text {leak }}}<0 .
$$

The overall effects of radiation induced changes in the $M_{F}$ threshold voltage and in the sensor leakage current are summarized in Fig. 6, showing the absolute value of the computed relative charge sensitivity variation $\frac{\Delta G_{Q, p}\left(\tau_{F}\right)}{G_{Q, p}}$ as a function of $\tau_{F}$. The relative charge sensitivity variation was obtained as $\frac{\Delta G_{Q, p}\left(\tau_{F}\right)}{G_{Q, p}}=\frac{G_{Q, p}\left(\tau_{F 0}\right)-G_{Q, p}\left(\tau_{F}\right)}{G_{Q, p}\left(\tau_{F 0}\right)}$, where $\tau_{F 0}$ is the preirradiation value of $\tau_{F}$ (about $110 \mu \mathrm{s}$ from simulations). Data points were obtained under the hypothesis that the shaper provides an ideal RC-CR filtering function and that the charge preamplifier yields a decaying exponential response to a delta-shaped pulse, as it has been assumed at the beginning of this section. The value of $\tau_{F}$ has been made to vary according to $g_{d s, F}$ values spanning between about $70 \mathrm{pA} / \mathrm{V}$ to $1000 \mathrm{pA} / \mathrm{V}$. Note that the absolute value of $\frac{\Delta G_{Q, p}\left(\tau_{F}\right)}{G_{Q, p}}$ is larger for larger peaking times. This is in agreement with the experimental results in Table III, showing the relative charge sensitivity variation measured in SRC and T1 type test structures after exposure to a $1100 \mathrm{krad}\left(\mathrm{SiO}_{2}\right)$ TID. Table III also shows that a larger relative gain change has been measured in SRC structures than in $\mathrm{T} 1$ samples, which are expected to feature a substantial sensor leakage current increase after irradiation. This is in agreement with the already discussed results of Fig. 4 and with (5), where $g_{d s, F}$ is demonstrated to decrease with increasing leakage current, therefore balancing the effect of $M_{F}$ threshold voltage shift and yielding a smaller gain change.

2) Radiation effects in the shaper: In the shaping stage, again the RINC effect can be invoked as the cause for charge sensitivity degradation. In particular, the NMOS current 


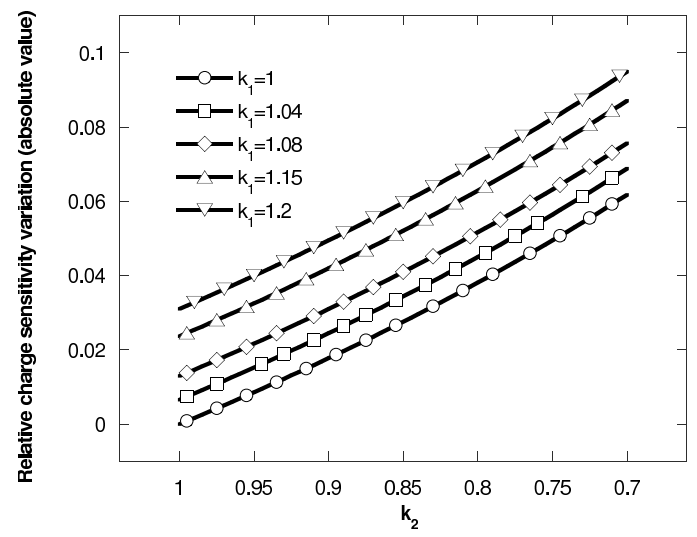

Fig. 7. Computed relative charge sensitivity variation as a function of the parameter $k_{2}$, plotted for different values of the parameter $k_{1}$.

sources (M1 to $M 4)$ in the transconductor, featuring a channel width of $0.25 \mu \mathrm{m}$, and the PMOS current source in the input stage, $M_{c s s}$, with $W=0.18 \mu \mathrm{m}$, may suffer a non negligible shift in their threshold voltage, due to charge trapping in the shallow trench isolation oxide. Circuit simulations show that a reduction in the threshold voltage of transistors $M 1$ to $M 4$ results in an increase in the current sunk by the sources, whose preirradiation value is $I_{t 0}$, and in a subsequent increase in the transconductance

$$
G_{m}=\frac{q k_{1} I_{t 0}}{2 n k_{B} T}=k_{1} G_{m 0},
$$

where the parameter $k_{1}>1$ takes into account the effects of radiation on $G_{m}$. On the other hand, a decrease of the threshold voltage in $M_{c s s}$ may be responsible for a decrease in the gain-bandwidth product $G B P$ through a decrease of the transconductance of the shaper input device

$$
G B P=\frac{k_{2} g_{m, i n s, 0}}{C_{z}}=k_{2} G B P_{0},
$$

where again $k_{2}<1$ accounts for radiation effects on the shaper input device transconductance. Assuming in this case for the charge preamplifier an ideal step response to a delta shaped pulse with $\mathrm{Q}$ area, it can be shown that the response of the overall channel takes the form

$$
v_{\text {out }, s}(t)=-2 \frac{C_{1} Q}{C_{F} C_{2}} \frac{e^{-\frac{2 k_{2} G m 0}{C_{2}} t}}{\sqrt{\left(\frac{k_{1}}{k_{2}}-1\right)}} \sin \left(\frac{t}{\tau_{1}}\right),
$$

where $\tau_{1}=\frac{C_{2}}{2 G_{m 0} \sqrt{k_{1} k_{2}-k_{2}^{2}}}$. Based on the definition given by the first equality in (2), the radiation induced relative variation of the charge sensitivity $\frac{\Delta G_{Q, s}\left(k_{1}, k_{2}\right)}{G_{Q, s}}=\frac{G_{Q, s}(1,1)-G_{Q, s}\left(k_{1}, k_{2}\right)}{G_{Q, s}(1,1)}$, has been computed and plotted, in the absolute value form, in Fig. 7 as a function of the parameter $k_{2}$ for different values of $k_{1}$. Note that the charge sensitivity decreases both for $k_{1}$ increasing and for $k_{2}$ decreasing.

\section{B. Equivalent noise charge}

Equivalent noise charge is a figure of merit for charge sensitive amplifiers. It is defined as the charge that has to be injected at the input of the charge measuring system in order to find a unit signal-to-noise ratio at its output [16]. The main noise sources in the DNW-MAPS under test are indicated in Fig. 1. The voltage source $e_{i n}$ represents the noise contribution from the preamplifier input device $M_{i n}$, whose power spectral density $\frac{d \overline{e_{i n}^{2}}}{d f}$ consists of a white component $S_{w s, i n}$, mainly accounting for channel thermal noise in the device drain current, and of a $1 / \mathrm{f}$ contribution $\frac{A_{f, i n}}{f^{\alpha} f n}$ :

$$
\frac{d \overline{e_{i n}^{2}}}{d f}=S_{w s, i n}+\frac{A_{f, i n}}{f^{\alpha_{f n}}}=n \gamma \frac{4 k_{B} T}{g_{m, i n}}+\frac{K_{f, i n}}{W L C_{o x} f^{\alpha_{f n}}} .
$$

In the previous equation, $\alpha_{f, n}$ is the $1 / \mathrm{f}$ noise slope coefficient, 0.85 in NMOS devices [17], $\gamma$ is the channel thermal noise coefficient, depending on the device operating point and polarity, $g_{m, i n}$ is the device transconductance and $K_{f, i n}$ is an intrinsic 1/f noise process parameter. The voltage source $e_{c s}$ represents the noise contribution from the PMOS current source in the preamplifier input branch. Its power spectral density $\frac{d \overline{e_{c s}^{2}}}{d f}$ features again a white component $S_{w s, c s}$ and a 1/f component $\frac{A_{f, c s}}{f^{\alpha f p}}$,

$$
\frac{d \overline{e_{c s}^{2}}}{d f}=S_{w s, c s}+\frac{A_{f, c s}}{f^{\alpha_{f p}}}=n \gamma \frac{4 k_{B} T}{g_{m, c s}}+\frac{K_{f, c s}}{W L C_{o x} f^{\alpha_{f p}}},
$$

where $\alpha_{f p}$ is the $1 / \mathrm{f}$ noise slope coefficient, between 1.05 and 1.20 for PMOSFETs, and $K_{f, c s}$ is an intrinsic $1 / \mathrm{f}$ noise process parameter depending on the overdrive voltage in PMOS transistors [17]. The preamplifier feedback MOSFET $M_{F}$ also provides a noise contribution, represented by the current source $i_{F}$, whose power spectral density $\frac{d \overline{i_{F}^{2}}}{d f}$, in the case of an NMOS transistor operated in deep subthreshold region, can be modeled as [18]

$$
\frac{d \overline{i_{F}^{2}}}{d f}=S_{w p, F}=2 q I_{D 0} e^{\frac{q\left(V_{G S}-V_{t h, F}\right)}{n K_{B} T}}\left(1+e^{-\frac{q V_{D S}}{k_{B} T}}\right) .
$$

The last noise contribution consists of a shot noise term in the sensor leakage current $I_{\text {leak }}$, modeled by means of the current source $i_{l}$ and featuring a power spectral density

$$
\frac{d \overline{i_{l}^{2}}}{d f}=S_{w p, l}=2 q I_{\text {leak }} .
$$

The above four noise terms yield for the ENC the expression given by (13) where $C_{T}$ is the total capacitance shunting the charge preamplifier input, including the detector capacitance $C_{D}$, the preamplifier input capacitance $C_{i n}$, the feedback capacitance $C_{F}$ and the injection capacitance $C_{i n j}$ (which, it is worth observing, has been included in the Apsel2T chip just for test purposes), while $A_{1}, A_{2}$ and $A_{3}$ are shaping coefficients. Some of the terms appearing in (13) exhibit a certain degree of sensitivity to ionizing radiation and are expected to feature a non negligible increase after exposure to $\gamma$-rays. Fig. 8 provides a typical example of the effects of a $1100 \mathrm{krad}\left(\mathrm{SiO}_{2}\right)$ TID on an NMOS transistor belonging to the same technology as the DNW-MAPS under test. The low frequency portion of the spectrum is clearly affected by irradiation, whereas white noise does not seem to undergo any sizeable degradation. Unlike what has been observed in 
$E N C^{2}=C_{T}^{2}\left\{A_{1} S_{w s, i n} \frac{1}{t_{p}}+A_{2} \cdot(2 \pi)^{\alpha_{f n}} A_{f, i n} t_{p}^{\alpha_{f n}-1}+\left[A_{1} S_{w s, c s} \frac{1}{t_{p}}+A_{2} \cdot(2 \pi)^{\alpha_{f p}} A_{f, c s} t_{p}^{\alpha_{f p}-1}\right] \frac{g_{m, c s}^{2}}{g_{m, i n}^{2}}\right\}+A_{3} t_{p}\left(S_{w p, F}+S_{w p, l}\right)$

$90 \mathrm{~nm}$ devices exposed to much higher doses [19], in the $130 \mathrm{~nm}$ NMOS under test, after the $100^{\circ} \mathrm{C} / 168 \mathrm{~h}$ annealing procedure, $1 / \mathrm{f}$ noise only partially recovers to its preirradiation level. This was actually found to be correlated to an incomplete recovery in the static characteristics, pointing to the fact that parasitic lateral transistors are still present. According to the model validated in previous works [20], [21], flicker noise performance degradation can be explained by means of the $1 / \mathrm{f}$ noise contributions coming from lateral parasitic devices turned on by positive charge buildup in the shallow trench isolation oxides (STI). The effect is larger in transistors with many fingers and operated at small current densities, where the parasitic lateral devices have a greater impact on the behavior of the main transistor. A minor, further contribution may be ascribed to border trap density increase in the main device gate oxide. The $1 / \mathrm{f}$ component of the noise voltage spectrum after irradiation $S_{f, p o s t}$ is therefore obtained by summing the contributions from the main transistor and from the equivalent parasitic transistor representing the effects of sidewall leakage in all the device fingers:

$$
S_{f, \text { post }}=\frac{\frac{K_{f, \text { main }}}{W L C_{o x}} g_{m, \text { main }}^{2}+\frac{K_{f, l a t}}{W_{l a t} C_{o x, l a t} L} g_{m, l a t}^{2}}{\left(g_{m, \text { main }}+g_{m, l a t}\right)^{2} f^{\alpha_{f n}}}
$$

In (14), $K_{f, \text { main }}$ and $K_{f, l a t}$ are postirradiation intrinsic process parameters for $1 / \mathrm{f}$ noise in the main device and in the equivalent parasitic transistor, featuring a transconductance $g_{m, \text { main }}$ and a transconductance $g_{m, l a t}$ respectively. For each transistor finger, 2 parasitic devices get switched on by radiation, each featuring a channel width $W_{l a t, f}$ and contributing to an equivalent parasitic transistor with channel width $W_{l a t}=2 n_{f} W_{l a t, f}, n_{f}$ being the number of fingers in the main device. The $C_{o x, l a t} W_{l a t, f}$ product, where $C_{o x, l a t}$ is the effective oxide capacitance of the parasitic STI sidewall transistor, can be extracted from static $I_{D}-V_{G S}$ curves [20]. The results from the rad-hard characterization of $130 \mathrm{~nm}$ NMOSFETs with different gate dimensions and operated at different drain currents were used to extract the main parameters appearing in (14). The extracted values, which are shown in Table IV, led to the theoretical evaluation of the noise degradation in the preamplifier input device, shown in Fig. 9. The relevant transconductances $g_{m, \text { main }}$ and $g_{m, l a t}$ were extrapolated from static measurements performed on devices operated at the same drain current density as $M_{i n}$.

TABLE IV

$1 / f$ NOISE PARAMETERS EXTRACTED FROM STM $130 \mathrm{~nm}$ NMOS DEVICES IRRADIATED WITH A $1100 \mathrm{krad}\left(\mathrm{SIO}_{2}\right) \gamma$-RAY DOSE.

\begin{tabular}{c|c|c}
$\begin{array}{c}W_{\text {lat }, f} C_{\text {ox }, \text { lat }} \\
{[\mathrm{fF} / \mu \mathrm{mm}]}\end{array}$ & $\begin{array}{c}K_{f, \text { main }} \\
{\left[10^{-25} \mathrm{JHz}^{-0.15}\right]}\end{array}$ & $\begin{array}{c}K_{f, l a t} \\
{\left[10^{-25} \mathrm{JHz} z^{-0.15}\right]}\end{array}$ \\
\hline $1.39 \cdot 10^{-10}$ & 20.0 & 11.9
\end{tabular}

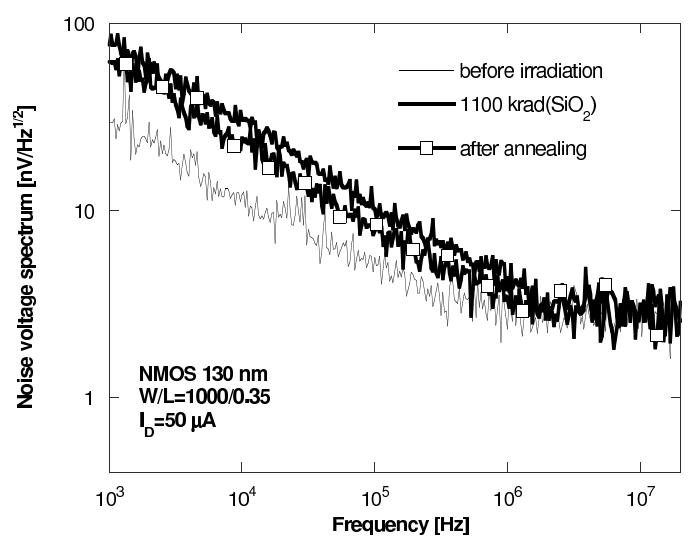

Fig. 8. Noise voltage spectra before and after exposure to $1100 \mathrm{krad}\left(\mathrm{SiO}_{2}\right)$ $\gamma$-ray TID and after annealing for an NMOS with $\mathrm{W} / \mathrm{L}=1000 / 0.35$ in the $130 \mathrm{~nm}$ technology at $I_{D}=50 \mu \mathrm{A}$.

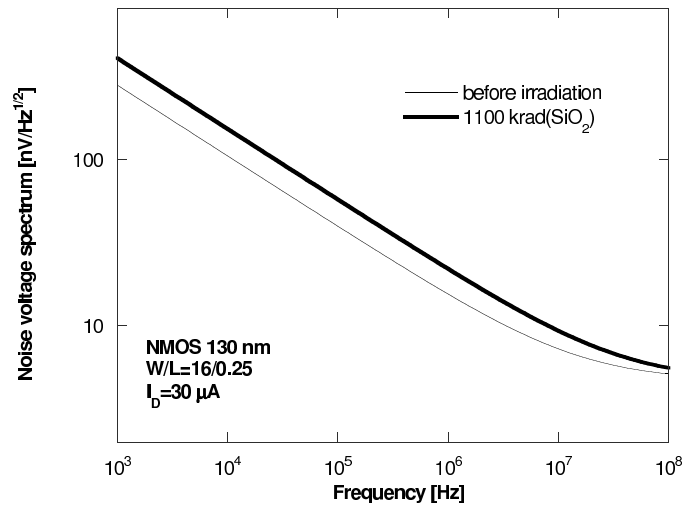

Fig. 9. Theoretical evaluation of the contributions to flicker noise degradation in the DNW-MAPS preamplifier input device.

Changes in the sensor leakage current and in the threshold voltage of the preamplifier feedback NMOSFET, which have already been discussed in the previous section, may be responsible for further ENC performance degradation in the MAPS devices. As far as $I_{\text {leak }}$ is concerned, an estimation of its increase after irradiation may be done by taking into account the results from irradiated DNW-diodes and suitably scaling their post-irradiation leakage current according to the ratio between the MAPS sensor perimeter and that of the DNW-diode test structures. The proposed estimation method, which yields $I_{\text {leak }}=16 \mathrm{pA}$ for MAPS of the T1 kind and $I_{\text {leak }}=20 \mathrm{pA}$ for MAPS of the T4 kind, is supported by the fact that due to the involved damage mechanism, i.e. charge trapping in the field oxide and creation of trapping states at the $\mathrm{Si} / \mathrm{SiO}_{2}$ interface over and around the junction, leakage current is expected to depend linearly on the length of the junction at the silicon surface. On the other hand, in order to determine the noise contribution from the preamplifier feedback MOSFET, its quiescent operating conditions (drain current and drain-to- 


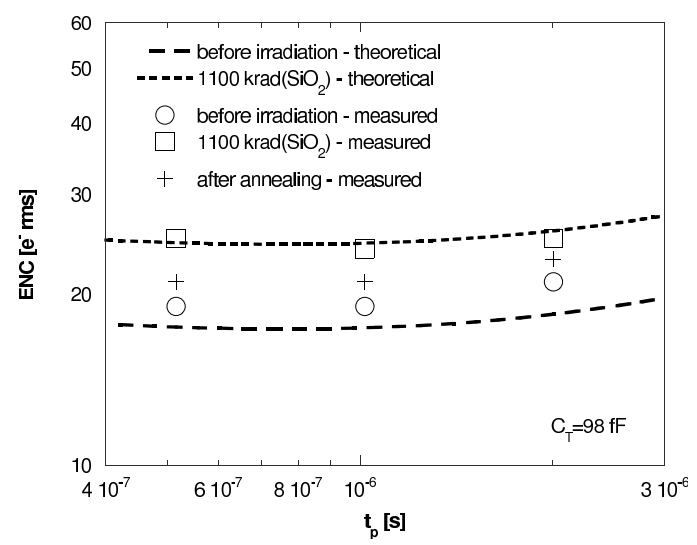

Fig. 10. Equivalent noise charge as a function of the peaking time for an SRC before irradiation, after exposure to a $1100 \mathrm{krad}\left(\mathrm{SiO}_{2}\right) \mathrm{TID}$ and after annealing. Experimental data are compared to theoretical evaluation based on (13).

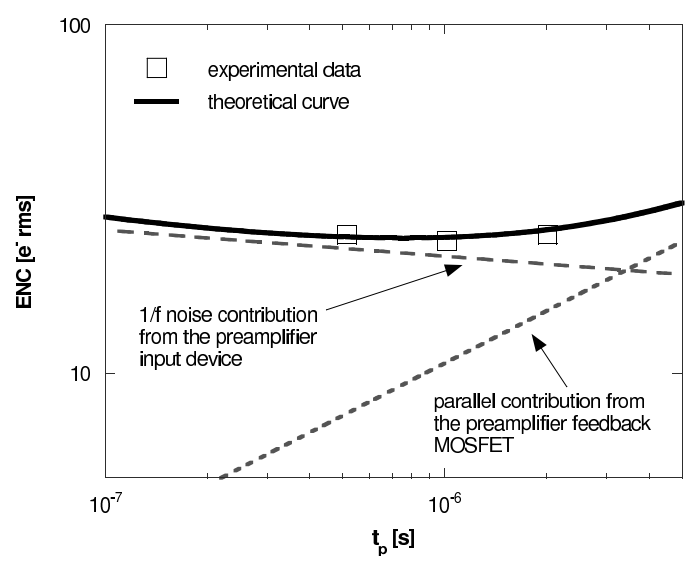

Fig. 11. Equivalent noise charge as a function of the peaking time for an SRC upon absorption of a $1100 \mathrm{krad}\left(\mathrm{SiO}_{2}\right)$. The main contributions to the theoretical ENC curve are shown.

source voltage) were extracted from circuit simulations.

Fig. 10 shows the equivalent noise charge as a function of the peaking time for an SRC before irradiation, after exposure to a $1100 \mathrm{krad}\left(\mathrm{SiO}_{2}\right)$ TID and after annealing. Experimental data are compared to theoretical evaluation based on (13). The ENC increase after irradiation is larger at short peaking times (about 25\%), mostly due to $1 / \mathrm{f}$ noise increase in the input device. However, at $t_{p}=2 \mu \mathrm{s}$, the parallel noise term from $M_{F}$ starts playing a significant role. This is apparent in Fig. 11, where the main contributions to the ENC in an SRC upon absorption of $1100 \mathrm{krad}\left(\mathrm{SiO}_{2}\right)$ are plotted. The total capacitance $C_{T}$ includes the injection capacitance ( $60 \mathrm{fF}$ ) and the preamplifier input (30 fF) and feedback capacitances ( $8 \mathrm{fF})$. Post-annealing partial recovery is likely to be correlated both to the observed annealing induced reduction in $1 / \mathrm{f}$ noise and to the shift of the threshold voltage of the preamplifier feedback MOSFET towards its original value.

Fig. 12 and Fig. 13 show the equivalent noise charge as a function of the peaking time before irradiation, after exposure to a $1100 \mathrm{krad}\left(\mathrm{SiO}_{2}\right) \mathrm{TID}$ and after annealing for the central pixel of a T3 matrix and for one of a T4 matrix respectively.

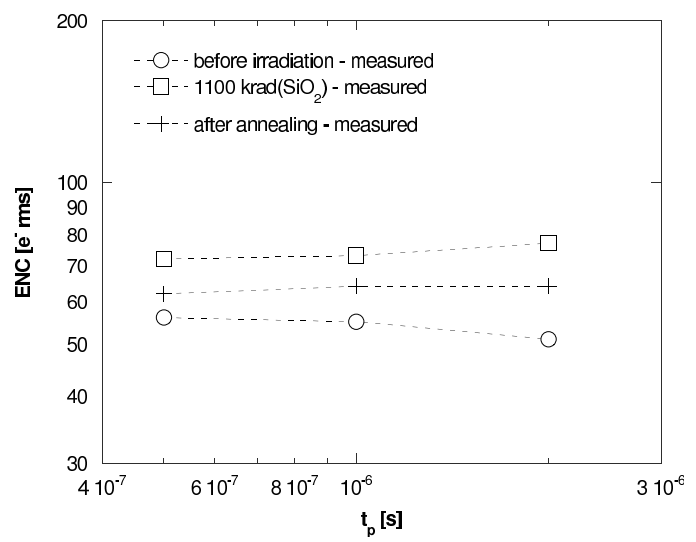

Fig. 12. Equivalent noise charge as a function of the peaking time for a sample of the T3 type before irradiation, after exposure to a $1100 \mathrm{krad}\left(\mathrm{SiO}_{2}\right)$ TID and after annealing.

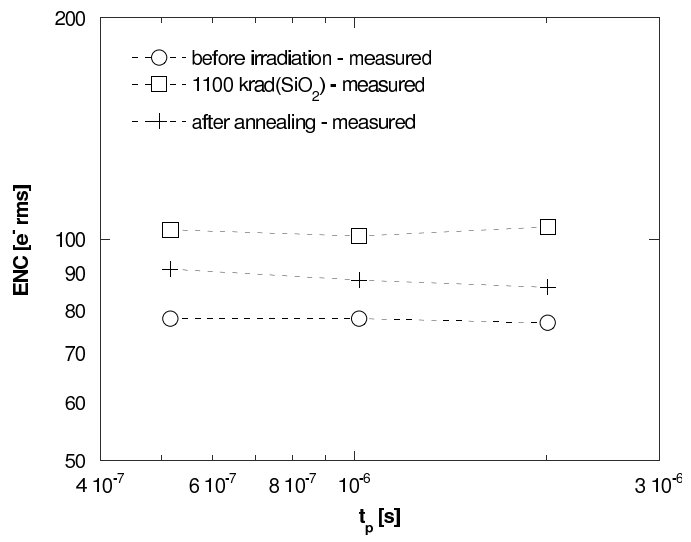

Fig. 13. Equivalent noise charge as a function of the peaking time for a sample of the $\mathrm{T} 4$ type before irradiation, after exposure to a $1100 \mathrm{krad}\left(\mathrm{SiO}_{2}\right)$ TID and after annealing.

In the pixel from the $\mathrm{T} 3$ structure, the slight change of slope after irradiation is related to the increase in the parallel noise contribution coming from the leakage current in the sensor and/or from the NMOS in the preamplifier feedback network, which have a major impact at larger peaking times. The ENC increase at $t_{p}=2 \mu \mathrm{s}$ is about $50 \%$, about $30 \%$ at $t_{p}=500 \mathrm{~ns}$. In the pixel from the T4 structure, the increase in ENC is not larger than $35 \%$ at all the peaking times. In this case, the relative weight of the noise contribution from the input device with respect to the noise from the sensor leakage current and from $M_{F}$ is larger than in the case of the T3 structure, due to the larger area and capacitance of the detector. After annealing, again, shift towards preirradiation ENC values can be observed, correlated to partial recovery in 1/f noise, sensor leakage current and $M_{F}$ threshold voltage. In the case of the T3 and T4 structures, the ENC model (the relevant curves are not shown in the figures) seems to significantly overestimate the experimental data both before and after irradiation. This might be due to inaccurate modeling of the collecting electrode capacitance in the process design kit which was considered for capacitance estimation. 


\section{CONCLUSION}

In this work, results from TID tests on a new type of sensor for particle tracking, based on a $130 \mathrm{~nm}$ deep $\mathrm{N}$ well CMOS technology, have been presented and discussed. Effects of $\gamma$-rays on the DUTs have been assessed by monitoring the evolution of charge sensitivity and equivalent noise charge with the integrated dose and after a $100^{\circ} \mathrm{C} / 168 \mathrm{~h}$ cycle. Reduction in charge sensitivity, never larger than $12 \%$ after $1100 \mathrm{krad}\left(\mathrm{SiO}_{2}\right)$ in the investigated devices, was found to originate mainly from radiation induced threshold voltage shift in narrow channel devices located in the feedback network of the charge preamplifier and in the shaper transconductor and input stage. Leakage current increase in the collecting electrode has been shown to have a role in balancing the effects from threshold voltage shift in the preamplifier feedback MOSFET. These results indicate that, in order to prevent excessive gain reduction, which may impact on fixed pattern noise performances in a multichannel system, particular care should be taken in avoiding narrow channel devices in some critical but well identified circuit points. Noise was found to feature a quite significant increase in deep $\mathrm{N}$-well MAPS exposed to $\gamma$-rays. Changes in a range from $25 \%$ to $50 \%$ with respect to preirradiation value were found after $1100 \mathrm{krad}\left(\mathrm{SiO}_{2}\right) \mathrm{TID}$. At short peaking times, the main source of degradation is to be found in the increase of low frequency noise in the preamplifier input transistor, as a consequence of charge trapping in the shallow trench isolation oxide running along the channel of the preamplifier input NMOS. Radiation induced leakage current increase in the collecting electrode and threshold shift in the preamplifier feedback, narrow channel MOSFET provides an explanation for ENC degradation at longer $t_{p}$ 's. This latter issue has already been addressed in a recently designed new version of the deep Nwell MAPS sensor by reducing the available peaking times according to the requirements of the experiments at the future high luminosity colliders. Hardening the preamplifier input transistor against TID may require minimizing the number of device fingers or using enclosed layout transistor (ELT) structures, respectively to reduce or completely avoid parasitic lateral paths along the STI sidewalls. Future plans include radiation hardness tests of the most recent versions of the deep $\mathrm{N}$-well MAPS detectors. Test structures with deep N-well, double junction diodes with different dimensions and geometry will be useful to better understand leakage current degradation under irradiation. Also sensitivity to bulk damage will be investigated through irradiation with protons and neutrons.

\section{ACKNOWLEDGMENT}

The authors wish to thank A. Faucitano (Università di Pavia), who made the ${ }^{60}$ Co source available for device irradiation, and M. Dellagiovanna, who carried out a large part of the measurements presented in this work. They are grateful to M. Giorgi (Università di Pisa) and his collaborators at INFN Pisa for their contribution to the development of the deep nwell sensor and for designing and providing the test diode structures. They are also indebted to V. Speziali (Università di Pavia, Italy) and L. Bosisio (Università di Trieste, Italy) for their useful suggestions.

\section{REFERENCES}

[1] I. Takayanagi, M. Shirakawa, K. Mitani, M. Sugawara, S. Iversen, J. Moholt et al., "A 1.25-inch 60-frames/s 8.3-M-pixel digital-output CMOS image sensor", IEEE J. of Solid-State Circuits, vol. 40, no. 11, pp. 2305-2314, Nov. 2005.

[2] Y. Degerli, M. Besanon, A. Besson, G. Claus, G. Deptuch, W. Dulinski, et al., "Performance of a Fast Binary Readout CMOS Active Pixel Sensor Chip Designed for Charged Particle Detection", IEEE Trans. Nucl. Sci., vol. 53, no. 6, pp. 3949-3955, Dec. 2006.

[3] M. Barbero, G. Varner, A. Bozek, T. Browder, F. Fang, M. Hazumi et al., "Development of a B-Factory Monolithic Active Pixel Detector The Continuous-Acquisition Pixel Prototypes", IEEE Trans. Nucl. Sci., vol. 52, no. 4, pp. 1187-1191, Aug. 2005.

[4] L. Ratti, M. Manghisoni, V. Re, V. Speziali, G. Traversi, S. Bettarini et al., "Monolithic pixel detectors in a $0.13 \mu \mathrm{m}$ CMOS technology with sensor level continuous time charge amplification and shaping", Nucl. Instrum. Methods, vol. A568, pp. 159-166, 2006.

[5] G. Traversi, M. Manghisoni, L. Ratti, V. Re, V. Speziali, "CMOS MAPS with pixel level sparsification and time stamping capabilities for applications at the ILC", Nucl. Instrum. Methods, vol. A581, pp. 291-294, 2007.

[6] A. Gabrielli, G. Batignani, S. Bettarini, F. Bosi, G. Calderini, R. Cenci et al., "Proposal of a data sparsification unit for a mixed-mode MAPS detector", 2007 IEEE Nuclear Science Symposium Conference Record, vol. 2, pp. 1471-1473, Oct. 26 2007-Nov. 32007.

[7] A. Besson, G. Claus, C. Colledani, Y. Degerli, G. Deptuch, M. Deveaux et al., "A vertex detector for the International Linear Collider based on CMOS sensors", Nucl. Instrum. Methods, vol. A568, pp. 233-239, 2006.

[8] SuperB, a High-Luminosity Asymmetric $e^{+} e^{-}$Super Flavour Factory. Conceptual Design Report.

http://www.pi.infn.it/SuperB/CDR.

[9] H. Spieler, Semiconductor Detector Systems, Oxford University Press, New York, 2005.

[10] L. Ratti, "Continuous Time Charge Amplification and Shaping in CMOS Monolithic Sensors for Particle Tracking”, IEEE Trans. Nucl. Sci., vol. 53, no. 6, pp. 3918-3928, Dec. 2006.

[11] R.J. Baker, H.W. Li, D.E. Boyce, CMOS circuit design, layout and simulation. The Institute of Electrical and Electronics Engineers, New York, 1998

[12] V. Re, M. Manghisoni, L. Ratti, V. Speziali, G. Traversi, "Total Ionizing Dose Effects on the Noise Performances of a $0.13 \mu \mathrm{m}$ CMOS Technology", IEEE Trans. Nucl. Sci., vol. 53, no. 3, pp. 1599-1606, Jun. 2006.

[13] F. Faccio, G. Cervelli, "Radiation-Induced Edge Effects in Deep Submicron CMOS Transistors", IEEE Trans. Nucl. Sci., vol. 52, no. 6, pp. 2413 2420, Dec. 2005.

[14] J. Bogaerts, B. Dierickx, G. Meynants, D. Uwaerts, "Total Dose and Displacement Damage Effects in a Radiation-Hardened CMOS APS", IEEE Trans. El. Dev., vol. 50, no. 1, Jan. 2003.

[15] E. Eid, T.Y. Chan, E.R. Fossum, R.H. Tsai, R. Spagnuolo, J. Deily et al., "Design and Characterization of Ionizing Radiation-Tolerant CMOS APS Image Sensors up to $30 \mathrm{Mrd}$ (Si) Total Dose", IEEE Trans. Nucl. Sci., vol. 48, no. 6, Dec. 2001.

[16] E. Gatti, P.F. Manfredi, "Processing the signals from solid-state detectors in elementary particle physics", La Rivista del Nuovo Cimento, vol. 9 , pp. 1-147, 1986.

[17] L.Ratti, M. Manghisoni, V. Re, V. Speziali, G. Traversi, "Minimum noise design of charge amplifiers with CMOS processes in the $100 \mathrm{~nm}$ feature size range", 2007 IEEE Nuclear Science Symposium Conference Record, vol. 4, Oct. 26 - Nov. 3 2007, pp. 2494-2502. Digital Object Identifier 10.1109/NSSMIC.2007.4436661.

[18] Y. Tsividis, Operation and modeling of the MOS transistor, McGrawHill, Boston, 1999.

[19] V. Re, L. Gaioni, M. Manghisoni, L. Ratti, V. Speziali, G. Traversi, "Comprehensive study of total ionizing dose damage mechanisms and their effects on noise sources in 90 nm CMOS technology", presented to the 2008 NSREC Conference, Tucson, Arizona July 14-18, 2008. To be published in the IEEE Trans. Nucl. Sci.

[20] V. Re, L. Gaioni, M. Manghisoni, L. Ratti, V. Speziali, G. Traversi, "Impact of Lateral Isolation Oxides on Radiation-Induced Noise Degradation in CMOS Technologies in the 100-nm Regime", IEEE Trans. Nucl. Sci., vol. 54, no. 6, pp. 2218-2216, Dec. 2007.

[21] L. Ratti, L. Gaioni, M. Manghisoni, G. Traversi, D. Pantano, "Investigating degradation mechnisms in $130 \mathrm{~nm}$ and $90 \mathrm{~nm}$ commercial CMOS technologies under extreme radiation conditions", IEEE Trans. Nucl. Sci., vol. 55, no. 4, pp. 1992-2000, Aug. 2008. 